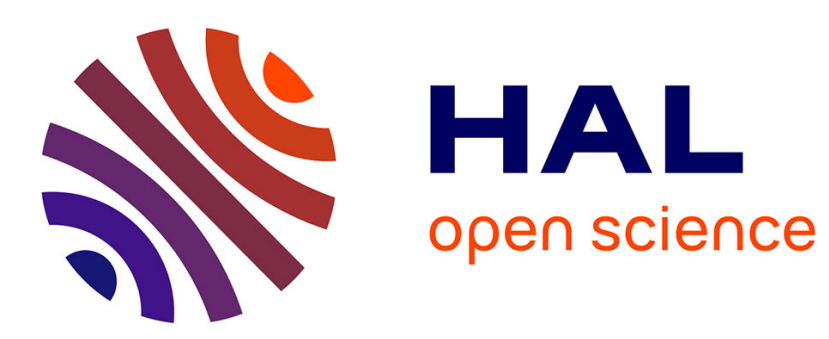

\title{
Etude épidemiologique du Marssonina brunnea (Ell. et Ev.) P. Magn
}

J. Pinon, Marie Poissonnier

\section{To cite this version:}

J. Pinon, Marie Poissonnier. Etude épidemiologique du Marssonina brunnea (Ell. et Ev.) P. Magn. European Journal of Forest Pathology, 1975, 5 (2), pp.97-111. 10.1111/j.1439-0329.1975.tb00451.x . hal-01594147

\section{HAL Id: hal-01594147 https://hal.science/hal-01594147}

Submitted on 2 Jun 2020

HAL is a multi-disciplinary open access archive for the deposit and dissemination of scientific research documents, whether they are published or not. The documents may come from teaching and research institutions in France or abroad, or from public or private research centers.
L'archive ouverte pluridisciplinaire $\mathbf{H A L}$, est destinée au dépôt et à la diffusion de documents scientifiques de niveau recherche, publiés ou non, émanant des établissements d'enseignement et de recherche français ou étrangers, des laboratoires publics ou privés. 
Pfeiffer, M., 1933: Frostuntersuchungen an Fichtentrieben. Tharandt. Forstl. Jahrb. 84, 664 bis 695 .

Pineau, M., 1968: Observations phénologiques et morphologiques sur le comportement de quelques essences forestières soumises à l'action des engrais N, P et K. Beih. Zeitschr. Schweiz. Forstver. 43.

Pisek, A.; Schiessi, R., 1947: Die Temperaturbeeinflußbarkeit der Frosthärte von Nadelhölzern und Zwergsträuchern an der alpinen Waldgrenze. Ber. Naturwiss.-med. Verein Innsbruck, 47, 33-52.

PüMPEL, B., 1973: Über die Auswirkungen von Düngung und Mykorrhizaimpfung auf Wachstum, Entwicklung, Mykorrhizabildung und Frostresistenz junger Fichten und Zirben. Diss. Universität Innsbruck.

SAKAI, A.; WeISER, C. J., 1973: Freezing resistance of trees in North America with reference to tree regions. Ecology 54, 118-126.

SANTARIUS, K. A., 1969: Die Wirkung von Salzen auf isolierte Spinatchloroplasten im Zusammenhang mit Problemen der Frost- und Trockenresistenz bei Pflanzen. Ber. dtsch. Bot. Ges. 82, 83-85

ScheumanN, W., 1962: Untersuchungen zur Frostresistenz der Douglasie (Psendotsuga taxifolia [Poir.] Britton) und Fichte (Picea abies [L.] Karst.). Deutsche Akad. Landwirtschaftswiss. Berlin. Tagungsberichte 53, 155-162.

Scheumann, W.; Hoffmann, K., 1967: Die serienmäßige Prüfung der Frostresistenz einjähriger Fichtensämlinge. Arch. Forstwes. 16, 701-705.

Tranquillini, W., 1958: Die Frosthärte der Zirbe unter besonderer Berücksichtigung autochthoner und aus Forstgärten stammender Jungpflanzen. Forstw. Cbl. 77, 89-105.

UlmER, W., 1937: Über den Jahresgang der Frosthärte einiger immergrüner Arten der alpinen Stufe, sowie Zirbe und Fichte unter Berücksichtigung von osmotischem Wert, Zuckerspiegel und Wassergehalt. Jb. wiss. Bot. 84, 553-573.

WeHrmanN, J., 1959: Methodische Untersuchungen zur Durchführung von Nadelanalysen in Kiefernbeständen. Forstw. Cbl. 78, 77-97.

- 1963: Möglichkeiten und Grenzen der Blattanalyse in der Forstwirtschaft. Landwirtschaftl. Forschung 16/2, 130-145.

Anschriften der Verfasser: Dr. BrigitTe PüMPel, dzt. Botanisches Institut der Hochschule f. Bodenkultur, A-1180 Wien, Gregor-Mendel-Straße 33; Dr. FriedeRike GöBl und Prof. Dr. Walter Tranquillini, Forstliche Bundesversuchsanstalt, Außenstelle f. subalpine Waldforschung, A-6020 Innsbruck, Hofburg, Rennweg 1

\title{
Etude épidemiologique du Marssonina brunnea (Ell. et Ev.) P. Magn.
}

\author{
Par J. Pinon et Marie Poissonnier
}

Réception du Ms. 10.5. 1974

\begin{abstract}
The epidemiology of Marssonina brunnea (Ell. et Ev.) P. Magn., a leaf parasite of cultivated poplars. The relationship between outbreaks of the disease and ripening of ascospores and release of conidia in spring was studied in 1972 and 1973 in the north (Lille) and the east (Nancy) of France.

During the growing season, the numbers of spores released by water splash during rainfall and also spores present in the air were systematically recorded. The various records and observations were related to climatic conditions.
\end{abstract}




\section{Introduction}

Le Marssonina brunnea (Ell. et Ev.) P. Magn., parasite de peupliers cultivés, entraine des réductions importantes de croissance et un affaiblissement des sujets sensibles, tel le Populus $x$ euramericana (Dode) Guinier cv. I-214, largement répandu en France. Les peupliers atteints par ce champignon sont caractérisés par la présence de taches brunes sur le limbe foliaire, les nervures principales, les pétioles et les jeunes rameaux. Le feuillage infecté jaunit progressivement et une défeuillaison précoce, responsable des dégâts, interyient alors.

Afin d'améliorer les méthodes de lutte opposables au Marssonina brunnea, nous avons tenté d'en préciser les modes de conservation et de dissémination en appréciant l'influence des facteurs climatiques sur ces phénomènes dans le Nord et l'Est de la France.

\section{Etude des sources d'infections primaires}

Dès 1965, les deux formes de conservation du Marssonina brunnea (Ell. et Ev.) P. Magn. étaient connues. Aux Pays- Bas, Gremmen insistait sur le rôle des ascospores dans le déclenchement des infections primaires, alors qu'en Italie, Castellani ne mettait en cause que les conidies. En 1968, Castellani et Freccero confirmaient l'existence des apothécies sur les feuilles tombées à terre et hivernant sur le sol de la peupleraie. Si, en Toscane, elles semblaient pouvoir jouer un certain rôle, dans le Nord de l'Italie, par contre, de nouvelles feuilles étaient déjà infectées alors que ces apothécies étaient encore immatures. Le rôle des ascospores étaient également mis en doute en Yougoslavie (Gojkovic 1970).

Les attaques du Marssonina brunnea étant d'autant plus dommageables qu'elles sont plus précoces et intenses, il nous est apparu nécessaire de connaître, dans les conditions climatiques du Nord (Lille) et de l'Est (Nancy) de la France, le rôle respectif des deux formes de conservation, afin de préciser les dates optimales d'intervention (lutte culturale et éventuellement chimique). Nous avons donc étudié en détail en 1972 et 1973, la maturation des ascospores et la libération des conidies dans les deux stations.

\subsection{Méthodes d'observation de la forme parfaite: Drepanopeziza punctiformis Gremmen}

\subsubsection{Détection des apothécies}

Des feuilles de peuplier présumées infectées et hivernant sur le sol de la peupleraie (Nancy) ou en pourrissoir (Lille) ont été récoltées chaque semaine, de novembre à juillet, et analysées au laboratoire. La détection des apothécies se pratique sous la loupe binoculaire (grosissement $25 \times$ ) après réhydratation des feuilles. Les fructifications apparaissent généralement groupées et plus nombreuses à la face inférieure des feuilles. Chaque récolte a fait l'objet d'un contrôle au microscope sur des coupes effectuées soit au microtome à congélation (Nancy) soit dans la paraffine (Lille) soit encore directement par écrasement (Nancy-Lille). Ceci nous a permis une détection plus fine des apothécies et une observation de l'évolution des stromas hivernant sur ces feuilles en fructifications sexuées.

\subsubsection{Contrôle de l'émission des ascospores}

Dès la première quinzaine de février, nous avons noté la présence d'apothécies renfermant asques et ascospores. Ceci ne préjuge en rien de leur état de maturité. Nous avons 
placé, après chaque récolte, des fragments de feuilles porteurs d'apothécies en cellules de van Tieghem; le fond de ces cellules est rempli de gélose à $2 \%$ afin de maintenir un taux d'humidité suffisant. Une lame vaselinée couvre ces cellules.

L'examen microscopique de cette lame permet de vérifier l'émission des ascospores. Ce dispositif est utilisé en projection ascendante et descendante (Fig. 1).

\subsubsection{Vérification du pouvoir pathogène des ascospores}

Une fois assurés de l'aptitude des asques à émettre leurs spores, nous avons cherché à contrôler leur pouvoir pathogène. Des feuilles porteuses d'apothécies sont collées sur le couvercle de grandes boîtes de Pétri où flottent des feuilles saines de Populus $x$ euramericana (Dode) Guinier cv. 1-214. A température du laboratoire (environ $20^{\circ} \mathrm{C}$ ), les taches apparaissent sur les feuilles saines après huit à dix jours (Fig. 1).

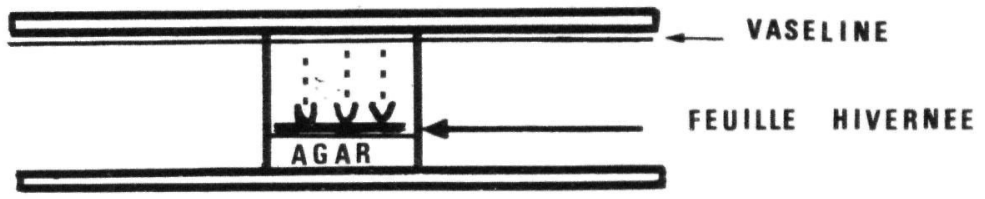

\section{controle des apothécies}

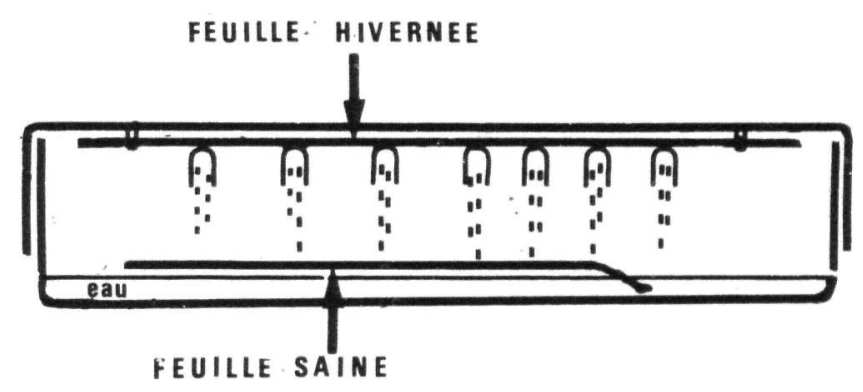

Fig. 1. Méthodes de contrôle de la maturation des apothécies et des ascospores

\subsection{Méthode d'observation de la forme imparfaite}

Une méthode simple de détection des conidies émises par les pustules de rameaux consiste à récupérer les eaux de pluie ruisselant sur un arbre infecté. Dans chaque station, nous avons choisi un sujet du clone 1-214. Une gouttière fixée sur le tronc guide les eaux de ruissellement que recueille un bidon à la base du fût. La technique d'installation du dispositif a été décrite par Aussenac (1968).

Après chaque pluie, le volume d'eau récupéré est mesuré et un échantillon en est prélevé, après agitation, pour filtration calibrée ( $1,2 \mu \mathrm{m}$ dans la plupart des cas). Le résidu solide (dont les conidies) retenu sur le filtre peut ensuite être mis en suspension dans une goutte de lactophénol pour dénombrement au microscope. Cette technique a l'inconvénient d'entraîner une relative imprécision du fait de la remise en suspension du résidu solide. Il est certes possible de rendre transparent le filtre sec (en l'imbibant d'huile à immersion). Malheureusement, les conidies du Marssonina brunnea sont hyalines et leur coloration sur filtre inégale. Cette technique, idéale pour les Dématiées, serait encore plus imprécise dans le cas présent. En pratique, il est possible de conserver les filtres, à sec, pendant plusieurs mois et donc de différer les comptages. Sur peuplier, les germinations dans l'eau de ruissellement sont très rares. Il peut en être autrement sur 
d'autres essences et la technique devra alors être modifiée (fongistatique dans le récipient). Le seuil des sensibilité de la méthode (pluie minimale pour permettre un ruissellement) est de l'ordre de 2 à $3 \mathrm{~mm}$ sur peuplier.

\subsection{Résultats}

\subsubsection{Evolution de la forme parfaite en 1972}

A Nancy, nous avons constaté l'emission d'ascospores mures dès la première semaine d'avril et jusque fin mai. Les émissions ont beaucop perdu en intensité à partir de la mi-mai.

A Lille, au cours de l'hiver, nous avons suivi la formation progressive des fructifications sexuées. A partir de décembre, l'observation de l'évolution de masses stromatiques plus ou moins immergées dans les tissus de la feuille nous a permis de constater la différenciation d'apothécies $(80-100 \mu \times 60-80 \mu)$ dans lesquelles nous avons distingué, à la mi-février de très nombreuses paraphyses et des asques contenant huit ascospores $(9-11 \mu \times 3-5 \mu)$.

Dans la première décade de mars, nous avons noté la présence de fructifications vides. Des ascospores avaient donc pu être émises avant cette date.

\subsubsection{Evolution de la forme parfaite en 1973}

A Nancy, les apothécies présentaient, dès février, des asques bleuissant à l'iode au sommet et contenant des ascospores. Toutefois, nous n'avons noté aucune émission de ces spores à cette époque. Celle-ci intervint début mars mais ne fut suivie d'infections notables (au laboratoire) qu'a la fin mars (récolte du 28 mars). L'émission et l'infection sont demeurées actives en avril et durant la première quinzaine de mai. Ensuite, l'intensité des émissions a diminué pour s'annuler fin mai, début juin.

A Lille, nous avons noté, dès le 12 février, la présence d'apothécies renfermant des asques à huit ascospores bien individualisées. Le premier contrôle de leur pouvoir pathogène effectué en mars a donné un résultat positif.

La production des fructifications sexuées est particulièrement abondante en mars et avril puis baisse progressivement pour s'annuler presque totalement début juin.

De plus, nous avons reçu régulièrement des feuilles hivernant en pépinière à Angers. Celles-ci furent, dès leur réception, contrôlées comme les autres récoltes. Lors du premier envoi (13 mars) tous les contrôles furent positifs. Nous avons noté la présence d'ascospores mûres jusque fin mai, début juin. Les premières taches étaient signalées au champ le 18 mai (communication personelle de M. RiDE). Ces observations sont en accord avec celles de Lille et de Nancy. Signalons qu'un échantillon provenant d'une parcelle traitée chimiquement comportait lui aussi de nombreuses apothécies.

\subsubsection{Evolution de la forme imparfaite en 1973}

Le Marssonina brunnea provoque sur les jeunes rameaux, de petites lésions ovales de 1 à $7 \mathrm{~mm}$ de long, brun-rouge, dont la partie centrale renferme de très nombreuses conidies agglutinées dans une substance mucilagineuse. Ces pustules conidiennes persistent pendant l'hiver.

L'analyse des eaux de pluie a permis de mettre en évidence plusieurs émissions de conidies avant le débourrement. Les spores ne peuvent donc provenir que des pustules de rameaux, d'ailleurs nombreuses dans le branches inférieures de l'arbre étudié.

Si certaines récoltes du mois d'avril n'ont révélé aucune spore, par la suite, toutes les pluies du mois de mai ont provoqué la libération des conidies. 


\subsubsection{Apparition de premières taches}

Comme nous le montre la fig. 2, pour le Nord et l'Est de la France, les deux types de spores (conidies et ascospores) sont présents lors du débourrement qui s'échelonne selon les clones et l'année entre la mi-avril et la mi-mai. Pour tenter de délimiter le rôle joué par ces deux formes de fructifications, il nous faut tenir compte des dates d'apparition des premières taches foliaires en peupleraie, des précipitations (nécessaires à la libération et à la germination des conidies) et de la durée d'incubation.

L'inoculation de feuilles saines par des ascospores, en étuve climatisée, sous 12 heures de lumière quotidienne nous a permis de préciser la durée d'incubation:

$8-12$ jours pour une température constante $\left(18-20^{\circ} \mathrm{C}\right)$

12 jours pour $19^{\circ} \mathrm{C}$ le jour et $7^{\circ} \mathrm{C}$ la nuit

16 jours pour $14^{\circ} \mathrm{C}$ le jour et $4^{\circ} \mathrm{C}$ la nuit

Ces valeurs sont très voisines de celles obtenues pour les conidies.

1973 _ VELAINE (EST)

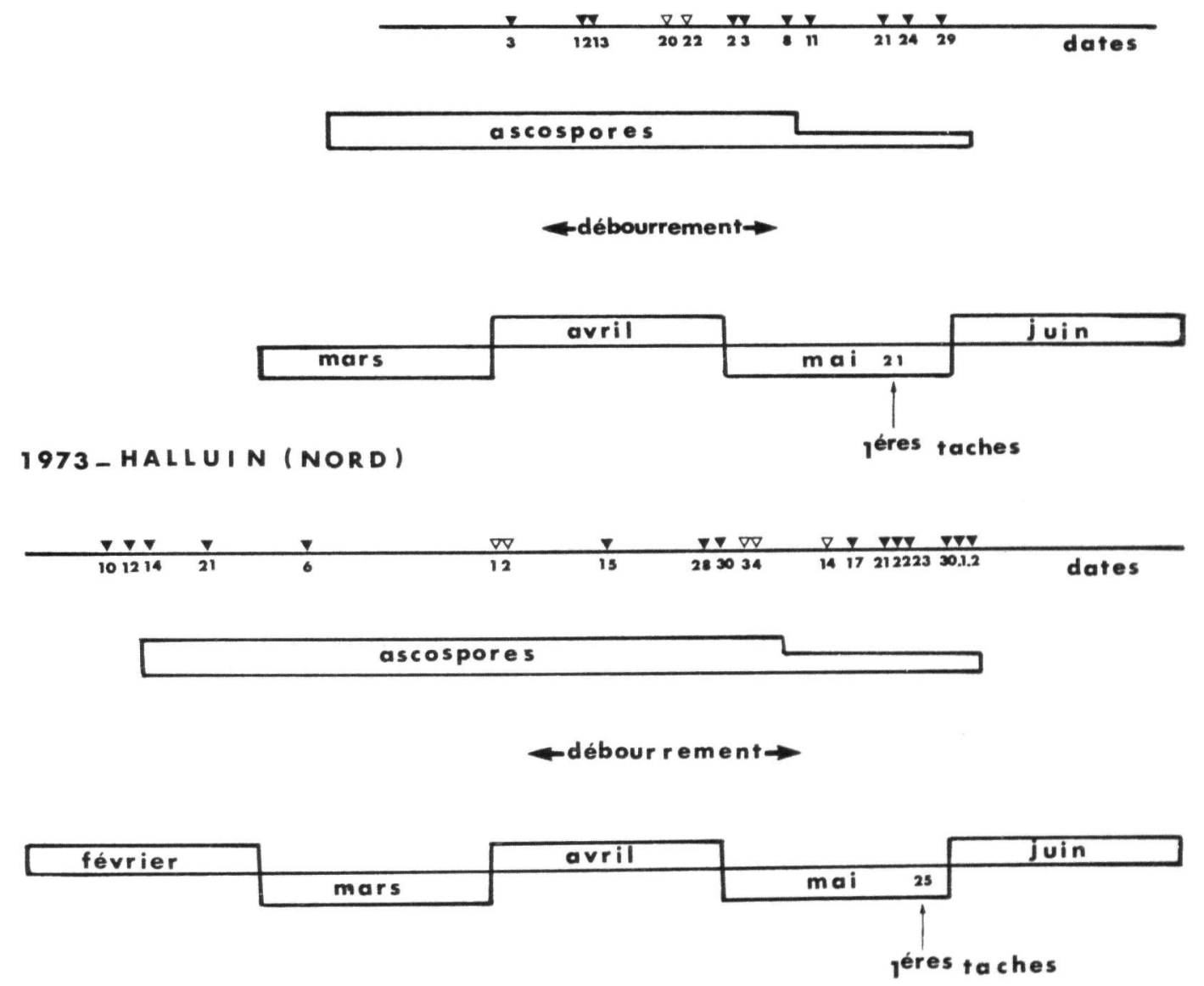

Fig. 2. Déclenchement des premières infections au printemps 1973 et mise en évidence des périodes d'émission des ascospores et des conidies issues des pustules de rameaux - Triangles noirs: pluies supérieures à $3 \mathrm{~mm}$ ayant libéré de nombreuses conidies; triangles clairs: pluies supérieures à $3 \mathrm{~mm}$ ayant libéré très peu de conidies ou pas du tout

\section{En 1972}

A Nancy, les premières taches ont été observées le 7 juin. Compte-tenu des températures enregistrées les jours précédents, la durée d'incubation a probablement été de 12 jours et les infections primaires ont donc eu lieu les 26-28 mai. Or, les ascospores étant libérées en petit nombre au cours de cette période, d'ailleurs abondamment pluvieuse, nous pouvons considérer que les infections primaires sont essentiellement le fait des conidies de rameaux. 
Dans le Nord, les premières taches brunes sur les feuilles sont apparues le 4 juin. Lors des jours précédents, la température moyenne a été de $9,5^{\circ} \mathrm{C}$ la nuit et de $15,5^{\circ} \mathrm{C}$ le jour et les précipitations abondantes. La durée d'incubation peut être estimée à une douzaine de jours ce que situe les infections primaires entre le 20 et le 23 mai. Or, à cette époque, les ascospores n'ont $\mathrm{pu}$ jouer qu'un rôle restreint dans les infections primaires qui sont donc essentiellement le fait des conidies des jeunes rameaux.

\section{En 1973}

A Nancy, les taches furent plus précoces (21 mai). Compte-tenu des températures, l'incubation a duré environ deux semaines. La date présumée d'infection se situe donc aux alentours du 7 mai. Cr des précipitations ont été enregistrées entre le 5 et le 9 mai, précédant une période assez sèche.

Des conidies avaient été captées assez nombreuses dans les eaux de pluie des 3 et 8 mai. A cette époque des ascospores mûres étaient encore émises.

A Lille, les premiers symptômes de la maladie sont apparus vers le 25 mai. Compte-tenu des moyennes de températures enregistrées les jours précédents $\left(9,1^{\circ} \mathrm{C}\right.$ la nuit et $14,2^{\circ} \mathrm{C}$ le jour) la durée d'incubation s'élève à 15 jours. Ainsi les infections primaires ont eu lieu aux alentours du 10 mai, date à laquelle les apothécies étaient encore détectées sur les feuilles, en quantité appréciable. A cette même époque, des conidies étaient récoltées dans les eaux de ruissellement. Ainsi en 1973, les deux types de fructifications du champignon ont pu jouer chacune un rôle dans les infections primaires, à Nancy comme à Lille.

\subsection{Discussion sur l'importance rélative des formes de conservation}

\subsubsection{Rôle de la forme sexuée}

Les ascospores libérées lors du débourrement (avril-mai) peuvent jouer un rôle non négligeable dans l'initiation des premières infections. Nous avons expérimentalement vérifié la sensibilité des jeunes tissus aux ascospores. L'infection peut avoir lieu dès la sortie de la première feuille. Les zones verte des écailles, mises à nu lors du gonflement du bourgeon, sont également sensibles. Par contre, les ascospores ne semblent pas aptes à infecter un rameau de un an bien aoûte.

Les études conduites sur les apothécies en cellules de van Tieghem ont montré que les asques étaient capables d'éjecter leurs spores sur une hauteur au moins égale à un centimètre. Celles-ci peuvent donc être entraînées à distance par le vent et assurer la propagation du parasite.

Castellani et Freccero (1970) ont proposé l'application d'une formule permettant de prévoir l'arrivée à maturité des apothécies. Nous avons tenté de l'appliquer aux conditions climatiques de 1972 et 1973.

Il apparait que l'émission des ascospores mûres aurait dû commencer le 3 avril en 1972 et le ler mai en 1973. Celles-ci furent décelées à Nancy début avril en 1972 et fin mars en 1973. Bien que deux années d'observations ne permettent pas de porter un jugement définitif, il semble que cette formule ne s'applique pas de manière satisfaisante dans nos conditions climatiques.

\subsubsection{Rôle de la forme asexuée}

En 1972 et 1973, les infections primaires ont eu lieu lors des périodes pluvieuses caractérisées par une température moyenne supérieure de $11^{\circ} \mathrm{C}$. Les conidies (qui sont libérées par la pluie) ont donc joué un rôle certain sous ces conditions climatiques. Ces spores furent détectées et dénombrées en 1973.

Rappelons que CASTELlani (1966) soulignait l'importance des pluies et de la température notant que le risque d'infection était nul au dessous de $8^{\circ} \mathrm{C}$. D'après nos observations, ce seuil de température semble pouvoir être relevé à $10^{\circ} \mathrm{C}$. Par contre, les 
pluie ayant ruissellé sur le feuillage d'un jeune sujet du clone Magister Géant fortement infecté. Une bonne concordance existe entre ces deux modes de détection. Plusieurs émissions importantes ont été simultanément enregistrées. Citons notamment les pluies du 8 et 16 août et du 10 septembre. La récolte du 16 août correspond à une forte précipitation orageuse $(48 \mathrm{~mm})$ et les deux autres à des pluies d'intensité moyenne mais survenant après une période de beau temps.

En 1973, chaque pluie de mars à octobre a fait l'objet d'un comptage de conidies. La fig. 3 récapitule l'ensemble des résultats. Trois périodes peuvent être distinguées dans l'année.

\subsubsection{Avant le débourrement}

Les premières dissémination de conidies sont apparues début avril (3-12 et 13). Les spores sont particulièrement nombreuses dans la pluie du $3(13,1 \mathrm{~mm})$ au contraire des deux autres récoltes. Signalons que cette émission a lieu à basse température (respectivement $3,1-3,9$ et $2,3^{\circ} \mathrm{C}$ ) et qu'aucun clone n'ayant de feuilles, elle ne peut avoir de conséquences notables, si ce n'est pour les conidies la possibilité d'atteindre les bourgeons et de contaminer ultérieurement les jeunes tissus verts dès leur apparition. Les autres pluies d'avril sont apparues «stériles».

\subsubsection{Du débourrement aux premières infections}

Chaque précipitation libère en mai des conidies dont le rôle dans le déclenchement des infections, a déjà été discuté. Fin mai-début juin, les spores deviennent rares. Or, à ce moment n'existe encore que très peu de taches sur les jeunes feuilles (les premières sont notées le $21 \mathrm{mai}$ ). Il semble donc que les stromas de rameaux cessent leur activité, un an (ou moins) après leur différenciation.

\subsubsection{Pendant la période de végétation}

De juillet à mi-octobre, chaque pluie libère un nombre variable de conidies provenant des acervules des feuilles et des rameaux verts. La principale cause de variation dans les effectifs rencontrés est la hauteur de précipitation. Le rôle de la température (généralement inférieure à $20^{\circ} \mathrm{C}$ ) est peu apparent. En première analyse, cette corrélation "précipitation-quantité de spores» est en accord avec les observations de CASTELLANI (1966). Il nous paraît toutefois essentiel (et les résultats de 1973 le confirment) de tempérer ce jugement par deux remarques restrictives. Les pluies de 23, 24 et 30 juillet, du 3 et 7 août, ont libéré moins de spores que prévu. Or, ces précipitations font suite à une série de pluies presque quotidiennes assez importantes (13 et 18 juillet, 30 août) qui avaient libéré de nombreuses spores. Il y a donc un effet de lessivage et il faut attendre près d'une semaine pour que de nouvelles spores apparaissent dans les acervules. Inversement, les pluies des 16 juillet, 18 août et 21 septembre sont très riches en spores. La hauteur des précipitations ne suffit pas pour rendre compte du pic d'émission du 19. Remarquons que cette pluie intervient après une période sèche assez longue (neuf jours sans pluie). Une méthode de prévision basée sur les données climatiques devra donc tenir compte de la répartition des pluies dans le temps.

Les conditions de dissémination des spores étant en partie précisées, se pose la question de leur efficacité dans la réalisation des infections. CASTELLANI (1966) fait remarquer que les températures moyennes élevées (supérieures à $20^{\circ} \mathrm{C}$ ) constituent un facteur limitant car elles favorisent l'évaporation et font donc obstacle à la germination des spores qui exige une période de «mouillé». En 1973, à Nancy, la température moyenne des journées pluvieuses est demeurée inférieure à $20^{\circ} \mathrm{C}$, sauf le 18 août $\left(21,5^{\circ} \mathrm{C}\right)$. 
Les relevés du pluviomètre nous ont permis d'évaluer pour la plupart des précipitations, leur «force» (quantité d'eau tombée par unité de temps). Il n'existe pas de relation nette entre ce critère et les effectifs récoltés.

Ces résultats sont confirmés par les comptages effectués à Lille en 1973 par quinzaine ou décade. Les émissions de conidies ont commencé un mois environ avant le débourrement pour cesser début octobre.

\section{Dissemination par voie aerienne}

Les premiers symptômes apparaissent toujours sur les branches les plus basses et gagnent progressivement la totalité du feuillage. Cette observation nous a fait supposer une dissémination à distance par voie aérienne.

\subsection{Méthode d'étude}

Nous avons utilisé des appareils similaires au capteur de spores de Hirst (Hirst 19521953). Notre but étant de préciser d'éventuelles corrélations entre les émissions de spores et les conditions climatiques, nous avons installé une station météo nous donnant la température, la pluviosité et le dégré d’hygrométrie.

L'étude a été faite dans une peupleraie de 1-214 âges de 6 ans (Lille) et de 14 ans (Nancy) pour une période s'étendant d'avril à novembre en 1972 et 1973.

\subsection{Résultats et discussion}

Les ascospores sexuées hyalines, unicellulaires de 3-5 $\mu \times 9-11 \mu$ n'ont pu être reconnues de façon certaine sur des lames vaselinées en raison de leur morphologie peu caractéristique et semblable à celles de nombreuses autres espèces saprophytes.

Déjà observées sur les feuilles attaquées et en culture, les conidies ont pu être assez facilement repérées sur les lames. Elles sont hyalines, de $12-15 \mu \times 5-7 \mu$, bicellulaires, la cellule supérieure étant deux fois plus longue que la cellule inférieure.

\subsubsection{En 1972}

A Lille, nous avons noté une émission du 19 août au 18 septembre, période où les arbres avaient déjà subi une chute importante de feuilles.

En établissant le graphique classique superposant les courbes de température, de dégré hygrométrique, de précipitations, de vitesse des vents et le nombre de conidies piégées, nous n'avons pu mettre en évidence aucune relation entre ces différents paramètres.

Par contre, nous avons pu observer l'existence d'un rythme journalier d'émission de conidies, elles ont lieu souvent la nuit entre $20 \mathrm{~h}$ et $8 \mathrm{~h}$ lorsque le degré hygrométrique est compris entre 85 et $100 \%$ et qu'il a accusé de fortes variations le jour précédent. Quant à la température, elle reste comprise entre 10 et $15^{\circ} \mathrm{C}$, en général. Il nous faut cependant noter qu'à Nancy comme à Lille les comptages n'ont jamais révélé de quantité très importante de conidies. 


\subsubsection{En 1973}

Dans l'Est (Nancy) et dans le Nord (Lille), nous n'avons pas constaté l'existence d'une période d'émission bien délimitée.

Dès la mise en place du capteur, un petit nombre de conidies a été piégé. Seules les pustules de rameaux pouvaient alors en être l'origine puisque les premières taches sur les feuilles ne sont apparues que fin mai.

Pendant la période végétative, la quantité journalière de spores est restée faible. Exceptionnellement, dans la nuit du 8 au 9 septembre, plusieurs milliers de conidies ont été captées (à Lille). A cette date, la seule donnée météorologique caractéristique est une pluie relativement abondante $(15,8 \mathrm{~mm})$ qui est survenue après une dizaine de jours de temps chaud et sec.

Le nombre de conidies n'apparaissant pas comme un paramètre significatif, nous avons préféré, pour les représentations graphiques, le critère du nombre des captures, ceci nous permettant de mieux préciser comment évolue la fréquence des émissions en fonction de la température, du degré hygrométrique et de l'heure.

Nous avons pu ainsi dégager les remarques suivantes:

- il existe une période semblable à un rythme circadien, les émissions de conidies ayant lieu préférentiellement de $19 \mathrm{~h}$ à $7 \mathrm{~h}$ sans toutefois s'annuler complétement aux autres moments de la journée (fig. 4);

- la dissémination présente un pic à 15 et $16^{\circ} \mathrm{C}$ et reste fréquente entre 11 et $18^{\circ} \mathrm{C}$. Les émissions sont toutefois relevées dans une gamme de température étendue $\left(9^{\circ} \mathrm{C}-25^{\circ} \mathrm{C}\right)$ (fig. 5$)$;

- le degré hygrométrique semble jouer un rôle non négligeable dans la dispersion des spores; les valeurs les plus favorables se situent entre 90 et $100 \%$ d'humidité relative et les conidies ne sont pratiquement pas capturées pour des valeurs inférieures à $70 \%$ (fig. 6).

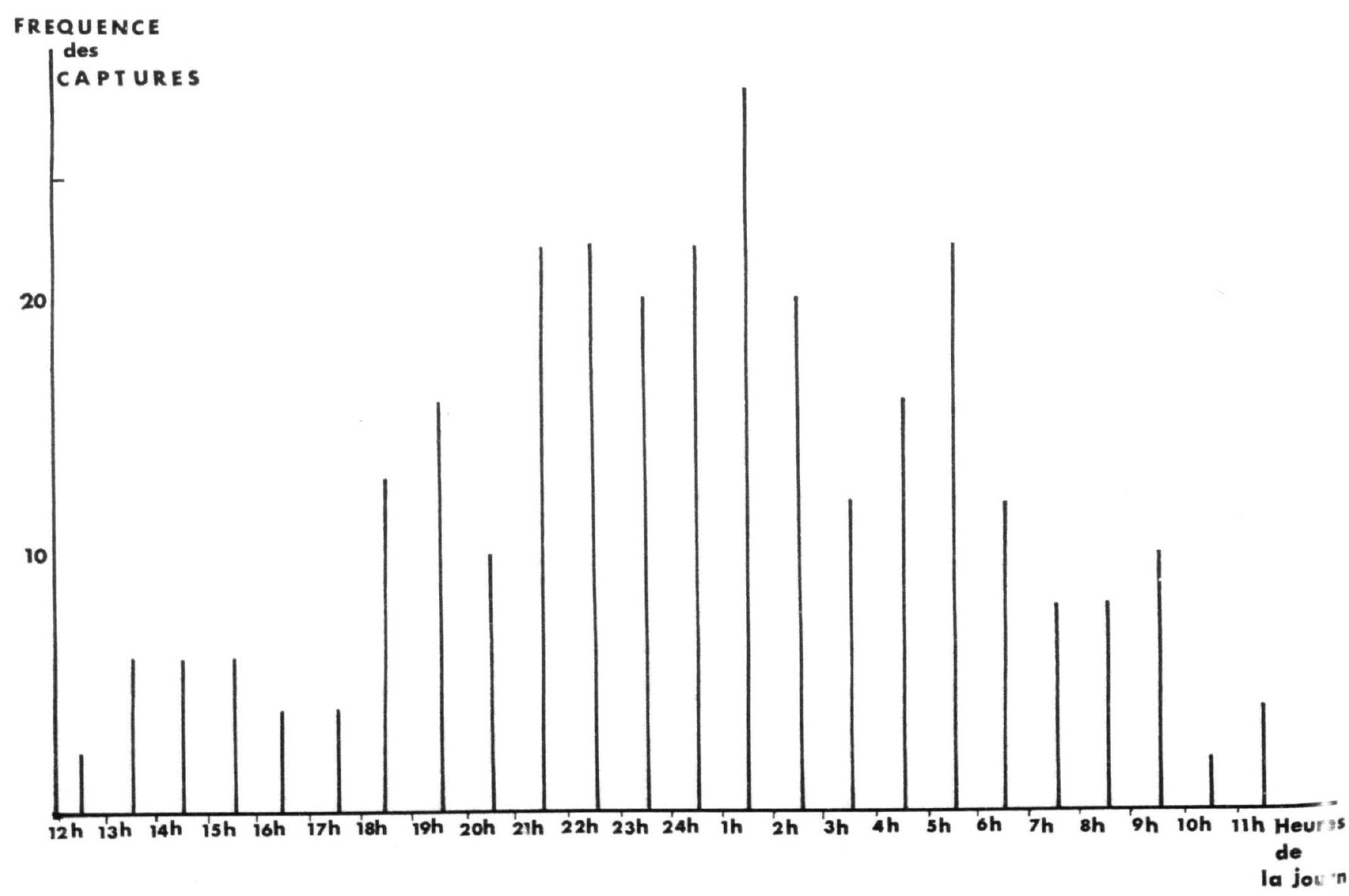

Fig. 4. Fréquence des captures aériennes de conidies selon l'heure de la journée au cours de la période complète d'observation de 1973 


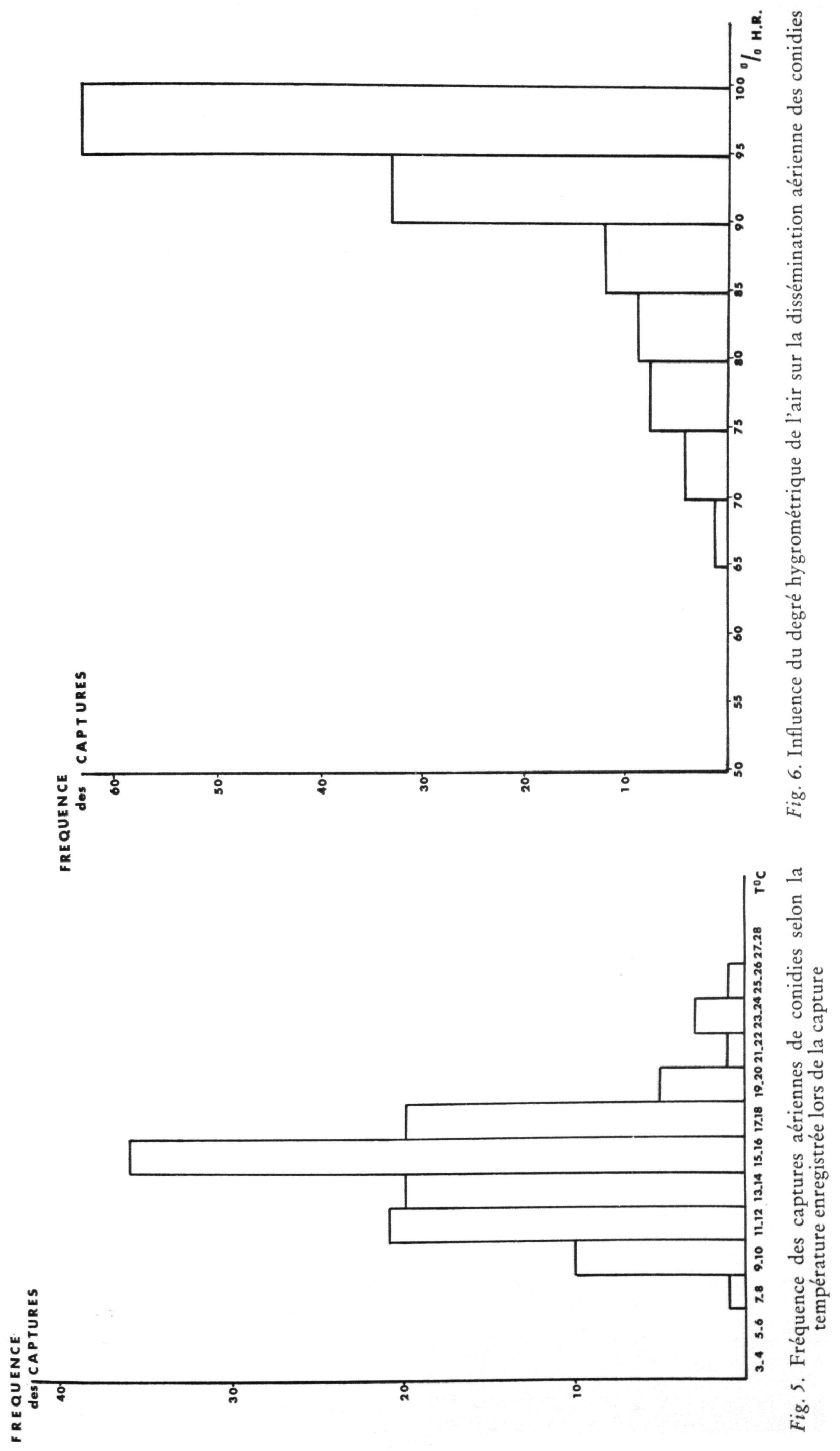


Si ces conditions de température et d'humidité relative sont en relation avec la libération des conidies, elles agissent également, d'après les travaux de CASTELLANI, sur la germination des spores et par là accélèrent le développement de la maladie.

Le capteur de Hirst n'ayant révélé que de faibles émissions de conidies, leur dispersion par voie aérienne ne semble donc pas être déterminante dans les infections, tout au plus joue-t-elle un rôle dans la propagation de la maladie d'arbre en arbre.

\section{Conclusions}

\section{Déclenchement des premières infections}

Les olservations pratiquées depuis deux ans dans les deux stations tendent à prouver que les deux formes de conservation du parasite sont actives lors du débourrement. Le rôle joué par chacune d'elles dépend de la date d'apparition des premières taches, donc du climat. Ainsi, le rôle des ascospores est-il plus important lorsque les infections sont précoces. La prévision météorologique à long terme étant imprécise, on devra lutter systématiquement contre ces deux formes par:

- l'enfouissement hivernal des feuilles tombées à terre, en travaillant le sol superficiellement (destruction des apothécies),

- l'élagage soigné (élimination partielle des pustules de rameaux des branches basses). En outre, le déclenchement des infections primaires devient prévisible. Dès le débourrement, il faut calculer la température moyenne des dix jours passés. Lorsque celle-ci dépasse $10^{\circ} \mathrm{C}$, il y a risque d'infection, si une pluie supérieure à $3 \mathrm{~mm}$ intervient. Ces conditions réalisées, on comptera sur une durée d'incubation variant entre 12 et 16 jours, selon la température, pour observer les premières taches. La prévision sera vérifiée

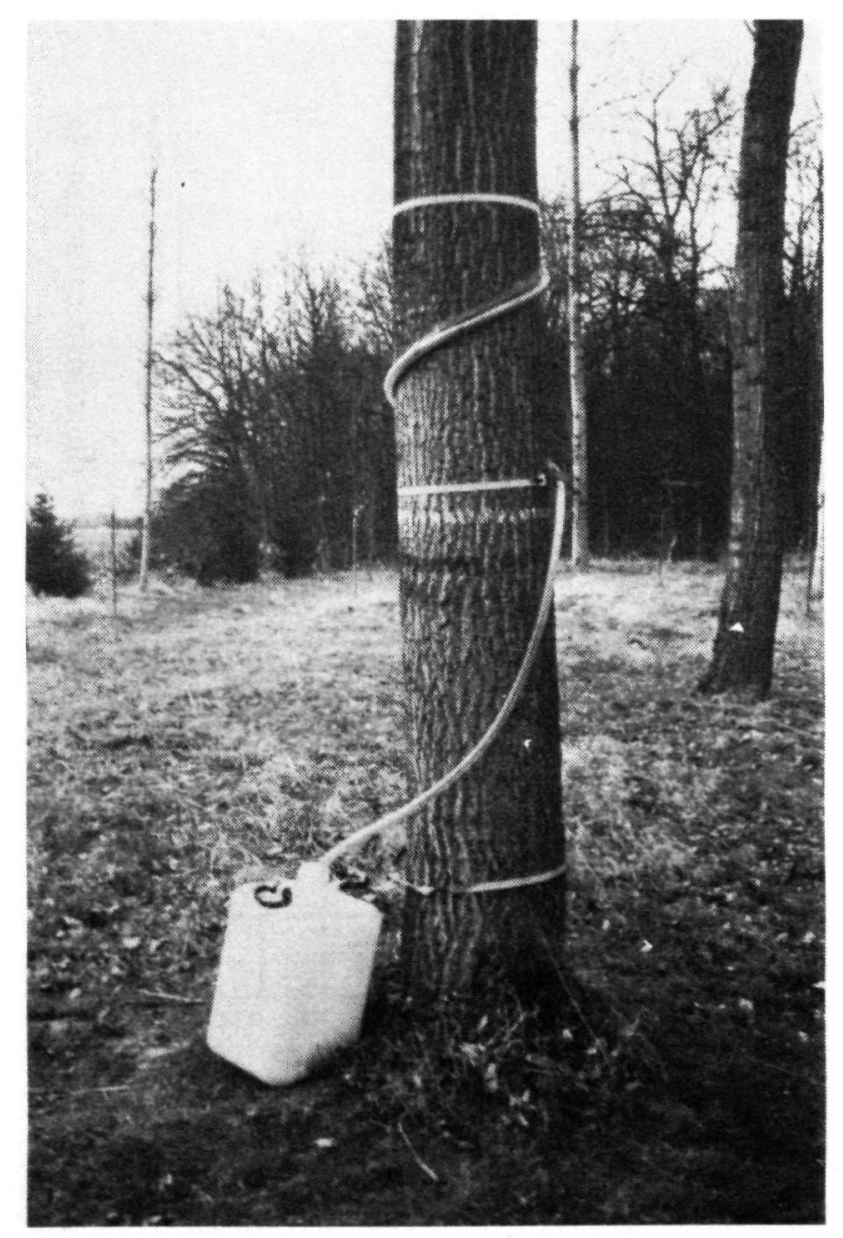
par le contrôle visuel en peupleraie. Lorsque les traitements chimiques s'imposent, il est essentiel de traiter dès l'apparition des premières taches.

Cette méthode pourra être affinée dans les prochaines années, lorsque nous aurons précisé certains points relatifs aux ascospores (conditions précises de dissémination, exigences pour la germination). Au contraire de nos collègues japonais (KoBAYASHI et CHIBA 1962) nous n'avons pas noté de manière appréciable la présence de conidies sur les feuilles tombées à terre.

Fig. 7. Dispositif en gouttière destiné à la récolte des eaux de pluie ruisselant sur l'arbre 


\section{Prévision des infections secondaires}

Les captures aériennes de conidies durant la période de végétation, bien que fréquentes, laissent apparaître des effectifs modestes comparés à ceux rencontrés dans les eaux de ruissellement. Leur incidence est donc faible et ne peut servir de base pour la lutte chimique. Malgré une relation évidente entre les captures aériennes et les données climatiques, l'absence ou la présence de spores demeurent difficiles à expliquer dans certains cas.

La dissémination aérienne des ascospores (au printemps) et des conidies (en été) permet de comprendre l'extension de la maladie vers le haut de l'arbre, ou de peupleraie à peupleraie (dissémination à distance). A l'inverse, la dispersion des conidies par les eaux de pluie explique l'accumulation de l'inoculum (donc des dégâts) dans la partie inférieure du houppier.

En 1966, Castellani mettait en évidence une relation entre les précipitations et les infections. Le dénombrement des conidies des eaux de ruissellement confirme ce résultat mais permet aussi d'appréhender des phénomènes biologiques plus fins. De ce fait, le barème de prévision des infections proposé par CAstellani (1966) ne peut être retenu sans tenir compte de la répartition des pluies dans le temps. Ainsi, les précipitations survenant après une période de temps sec constituent un risque élevé d'infection malgré une faible pluviosité décadaire. Au contraire, les dernières pluies d'une période pluvieuse sont peu dangereuses, les stromas ayant été vidés de leurs spores, même si le bilan décadaire de la pluviosité est élevé. Nous avons poursuivi cette analyse en appliquant le barème de Castellani aux données climatiques de Nancy pour trois années de référence, à savoir 1966 (forte attaque), 1967 (attaque d'intensité moyenne) et 1973 (attaque faible). Les résultats obtenus figurent au le tableau. Si la précocité des attaques ressort assez nettement, les prévisions estivales ne rendent pas pleinement compte des différences d'évolution des attaques au cours de ces trois années. Nous pensons que

\section{Tablean}

Estimation du risque d'infection a Nancy selon le bareme de Castellani

\begin{tabular}{|c|lll|}
\hline Décade & \multicolumn{1}{|c|}{1966} & \multicolumn{1}{c|}{1967} & 1973 \\
\hline $1-10$ avril & nul/bas & nul & nul \\
$11-20$ avril & bas & nul & nul \\
$21-30$ avril & bas & nul & nul \\
$1-10$ mai & bas & nul/bas & moyen \\
$11-20$ mai & bas & moyen & bas \\
$21-31$ mai & nul/bas & bas & moyen \\
$1-10$ juin & nul & nul & nul \\
$11-20$ juin & élevé & nul & moyen \\
$21-30$ juin & élevé & nul & nul \\
$1-10$ juillet & moyen & moyen & nul \\
$11-20$ juillet & nul & nul & moyen/élevé \\
$21-31$ juillet & nul & nul & moyen/élevé \\
$1-10$ août & élevé & moyen/élevé & moyen/élevé \\
$11-20$ août & moyen & élevé & nul \\
$21-31$ août & bas & nul & nul \\
$1-10$ septembre & moyen & bas/moyen & nul \\
$11-20$ septembre & nul & bas & élevé \\
$21-30$ septembre & nul & élevé & nul \\
\hline
\end{tabular}


cette imprécision est liée au fait que ce barème décadaire ignore le régime des précipitations (répartition et espacement dans le temps). De plus, notre étude utilise une méthodologie différente sous un climat peu comparable à celui de la vallée du Pô.

\section{Rémerciements}

Nous tenons à remercier Monsieur le Professeur Lacoste de la Faculté des Sciences et Techniques de Lille, l'Institut de Développement Forestier, Monsieur Louvegnies, Ingénieur du Groupement de Gestion et de Production forestière du Nord.

Nous associons à nos remerciements Madame ArLette Schipper pour son aide dévouée.

\section{Résumé}

Au printemps, nous avons contrôlé chaque semaine, pour deux stations, la maturation des ascospores naissant sur les feuilles tombées à terre. Simultanément, l'émission des conidies issues des pustules de rameaux et libérées par les pluies a été étudiée, avant et après le débourrement. Le champignon, conservé sous ses deux formes est en activité lorsque les jeunes feuilles apparaissent. Le rôle joué par chacune d'elles dans le déclenchement des infections primaires est sous la dépendance des conditions climatiques. Ces infections interviennent lorsque la température moyenne dépasse $11^{\circ} \mathrm{C}$ et si une pluie surgit; la durée d'incubation varie, selon la température, entre 12 et 16 jours.

Pendant la saison de végétation, chaque pluie libère des spores mais leur nombre est très variable. Ces récoltes sont particulièrement nombreuses si une forte pluie survient après une semaine de beau temps. En juin, les pustules de rameaux de l'année précédente cessent leur activité et peu de spores sont récoltées car les taches sont encore peu nombreuses sur les jeunes feuilles et rameaux. Pour le Nord et l'Est de la France, la méthode de prévision des infections proposée par Castellani est valable sauf dans deux cas qui sont discutés.

L'utilisation d'un capteur type Hirst a permis de mettre en évidence des émissions quasiquotidiennes de spores pendant les périodes humides (plus de $90 \%$ d'humidité relative) Leur rôle dans les infections paraît modeste si nous les comparons aux effectifs rencontrés dans les eaux de ruissellement.

\section{Summary}

During spring, ripening of ascospores from fallen leaves was recorded weekly for two years at two stations. At the same time, spores from pustules on twigs, before and after flushing were collected after rainfall. Both spore types were present at the time of flushing. Climatic conditions are important for both types in influencing the amount of infection. Primary infections occur when the mean temperature exceeds $11^{\circ} \mathrm{C}$ and apparently when rainfall has occurred. Incubation lasts for 12 to 16 days, according to temperature.

During the growing season, spores are released after each rainfall, but the number of spores trapped varies considerably. It is very high after heavy rainfall or, whatever the rainfall, when it occurs after a week of dry weather. In June, old twig pustules cease activity and few spores are collected, because young leaf and twig pustules are still scarce. In the north and east of France, the method of forecasting infection proposed by CASTELLANI appears to be of value, except in two cases which are discussed.

Spore discharges were detected almost every day by mean of Hirst's spore trap during periods when the relative humidity exceeded $90 \%$. Spores released in this way seem to play a less important role than spores released after rainfall.

\section{Zusammenfassung}

\section{Die Epidemiologie von Marssonina brunnea, einem Blattparasiten an Wirtschaftspappeln}

In wöchentlichen Abständen wurde während des Frühlings der Reifungsprozeß der Ascosporen auf abgefallenen Blättern an zwei Untersuchungsstationen verfolgt. Während der gleichen Zeit wurden nach Regenfällen Sporen gesammelt, welche teils vor, teils nach dem Austrieb aus Sporenlagern an Zweigen stammten; beide Sporenformen des Pilzes waren aktiv. Die Bedeutung beider Sporenarten für den Ausbruch der Krankheit hängt von den klimatischen Bedingungen ab. Primärinfektionen finden statt, wenn das Temperaturmittel $11^{\circ} \mathrm{C}$ übersteigt und anschei- 
nend sind Niederschläge vonnöten. Die Inkubationszeit beläuft sich auf etwa 2 Wochen, 12 bis 16 Tage, je nach der herrschenden Temperatur.

Während der Wachstumsperiode werden nach jedem Regen Sporen entlassen, die Zahl der aufgefangenen Sporen schwankte jedoch erheblich. Sie ist besonders hoch nach starken Niederschlägen oder - unabhängig von der Intensität der Niederschläge - wenn der Regen nach einer einwöchigen Trockenperiode fällt. Im Juni stellen die alten Sporenlager auf den Zweigen ihre Aktivität ein und es werden nur wenige Sporen aufgefangen, weil es zu dieser Zeit nur wenige Lager auf Blättern und Zweigen gibt. Im Norden und Osten Frankreichs scheint die von $\mathrm{CA}_{\mathrm{A}}$ STELLANI vorgeschlagene Methode der Befallsprognose von Nutzen zu sein, mit Ausnahme zweier näher diskutierter Fälle.

Bei Perioden feuchten Wetters (rel. Feuchte über 90\%) wurden mit Hilfe der Hirstschen Sporenfalle fast täglich Sporenentlassungen registriert. Sie scheinen jedoch - verglichen mit den Sporenentlassungen nach Regen - keine wesentliche Rolle für die Infektion der Pappel zu spielen.

\section{Bibliographie}

Aussenac, J., 1968 Interception des précipitations par le couvert forestier. Ann. Sci. Forest. 25, $135-136$.

CASTEllani, E., 1965 : Influence des facteurs climatiques sur les infections des peupliers euraméricains par Marssonina brunnea. Groupe de travail des maladies. Comm. Int. du Peuplier Wageningen 7-9-IX-1965.

- 1966: Influence des facteurs climatiques sur les infections des peupliers euraméricains par Marssonina brunnea (Ell. et Ev.) P. Magn. Phytopath. médit. 5, 41-52.

Castellani, E.; Freccero, V., 1968: Differenziazone dell'apotecio di Drepanopeziza punctiformis Gremmen in condizioni climatiche diverse. Riv. Patol. Veg. Pavia 4, 151-164.

- - 1970: Further observations on the ripening of the ascospores of Drepanopeziza punctiformis. Working group on disease. International Poplar Commission Farnham, 1970.

Gojkovic, N., 1970: Nouveaux résultats dans la recherche sur le Marssonina brunnea (Ell. et Ev.) P. Magn. en Yougoslavie. Groupe de travail des maladies. Comm. Int. du Peuplier Farnham, 1970.

Gremmen, J., 1965: L'infection du Peuplier par le champignon Marssonina. Groupe de travail des maladies. Comm. Int. du Peuplier Wageningen, 1965.

Hirst, J. M., 1952: An automatic volumetric spore trap. Ann. Appl. Biol. 39, 257-265.

- 1953: Changes in atmospheric spore content, diurnal periodicity and the effects of weather. Trans. Br. Mycol. Soc. 36, 375-393.

Kobayashi, T.; Chiba, O., 1962: Overwintering of some leaf attacking fungi of poplars. Jour. Jap. For. Soc. 44, 19-24.

l'Adresse des auteurs: J. Pinon, Laboratoire de Pathologie forestière C. N. R. F., F-54370 Champenoux-Einville; Marie Poissonnier - AFOCEL, Domaine de l'Etançon, F-77730 - Nangis 
This document is a scanned copy of a printed document. No warranty is given about the accuracy of the copy. Users should refer to the original published version of the material. 